the diagnosis and treatment of ear disease as well as the longer term aim to identify and train a Malawian otologist. The project has also raised awareness of deafness in Malawi and has highlighted the significant public health issues relating to the aetiology of ear disease and deafness in $3^{\text {rd }}$ world countries.

As well as addressing the practical and ethical issues relating to the project the presentation will outline future plans in developing both implant and otological surgery in Malawi and southern Africa.

doi:10.1017/S0022215116003236

\section{Chronic Ear Diseases in developing world (R744)}

\section{ID: 744.2}

What do we know about the burden of cholesteatoma in the developing world and what strategies could help

\section{Presenting Author: Michael Smith}

Michael Smith

International Nepal Fellowship

Learning Objectives: Understanding the burden of cholesteatoma in the developing world. Consideration of optimum management strategies in resource poor settings.

Chronic Otitis Media and its effects include hearing loss, reduced Quality of Life (QoL) and life threatening complications. These are major public health problems in developing countries. Many reviews include data from small or old studies and make generalisations that may now be inaccurate. The prevalence of cholesteatoma in most developing countries is unclear. It appears to be less frequent in some populations such as parts of Africa and S America, but much commoner in others such as SE Asia. Understanding of Otitis Media and $\mathrm{COM}$ has taken major steps forward in recent years. Risk factors and causes are generally agreed, but those specifically for cholesteatoma are less clear, in a developing country context. Some factors are amenable to targeted public health and primary care interventions and some countries have seen reducing incidence of COM and its complications. Prevalence studies of COM rarely distinguish between types of COM such as mucosal central perforations and cholesteatoma. Often the skills and materials required for diagnosis are lacking in under resourced health systems. Most agree that the treatment of cholesteatoma requires surgery. The complications of cholesteatomatous COM are usually considered more severe than mucosal COM. Both can be life threatening and many cases of 'safe' COM can also benefit from surgery. In poor resource settings with few specialists, how can patients be identified and surgery delivered? Do out reach camps play a useful part? Extensive disease is common and late stage, often worse than commonly seen by specialists from developed centres. What forms of surgery are most cost effective and safe to teach? Can developed nations partner in the development and training of local specialists?

After over 30 years experience in such settings, principally in Nepal I hope to open up some of these questions. doi: $10.1017 / \mathrm{S} 0022215116003248$

\section{Chronic Ear Diseases in developing world (R744)}

\section{ID: 744.3}

The impact of chronic otitis media on quality of life

\section{Presenting Author: Robin Youngs}

Robin Youngs ${ }^{1}$, Edward Maile ${ }^{2}$, Puran Tharu ${ }^{3}$, Hannah Blanchford ${ }^{4}$, Rachel Edmiston ${ }^{5}$

${ }^{1}$ Gloucestershire Hospitals NHS Foundation

Trust, ${ }^{2}$ Harvard University, ${ }^{3}$ Britain Nepal

Otology Service (BRINOS), ${ }^{4}$ ENT Department,

Darlington Memorial Hospital, ${ }^{5}$ University

Hospital of South Manchester

Learning Objectives: To present quality of life issues in chronic otitis media, including the impact of corrective surgery. This study focuses in chronic otitis media in a developing world setting.

Hearing impairment is a significant burden in the developing world. However, no suitable quality of life (QoL) measures exist for use in Nepal. We aimed to amend and translate the Glasgow Health Status Inventory (GHSI), assessing QoL at any given time, and the Glasgow Benefit Inventory (GBI), assessing change in QoL following intervention, into Nepali and to assess the impact of ear disease and effect of surgery on QoL.

The GHSI and GBI were translated into Nepali and independently verified. The GHSI was administered by interview to patients before surgery, and the GBI was administered 6 months after surgery. The Mann-Whitney U-test was used for hypothesis testing.

The GHSI was administered to 242 patients. In total, 205 had chronic suppurative otitis media (CSOM) without cholesteatoma and 37 had cholesteatoma. The mean GHSI score was 47.9.

There was no significant difference in GHSI scores between patients with CSOM without cholesteatoma and those with cholesteatoma. The GBI was administered to 161 patients, 73 of whom had also been in the GHSI group. In total, 130 had CSOM without cholesteatoma, 31 had cholesteatoma. The mean GBI score was +38.4 with no significant difference between disease groups.

Conclusions: Ear disease in Nepal is associated with reduced QoL, and surgical intervention is associated with improved QoL. There is no difference in QoL or benefit following surgery for CSOM between patients with or without cholesteatoma. There are few QoL measures suitable for the developing world. It is essential to invest in these measures to guide health interventions.

doi:10.1017/S002221511600325X

Chronic Ear Diseases in developing world (R744)

\section{ID: 744.4}

Chronic Ear Diseases in The Developing World

Presenting Author: Mohan Kameswaran 